\title{
A TECHNIQUE FOR OFDM SYMBOL SLICING
}

\author{
Ana Perez-Neira Miguel A. Lagunas \\ Universitat Politècnica de Catalunya (UPC)/Centre Tecnològic de Catalunya (CTTC- CERCA) \\ Av. Carl Friedrich Gauss 7, 08860 Castelldefels, Spain
}

\begin{abstract}
This work presents an orthonormal transform that splits the Orthogonal Frequency Division Multiplex (OFDM) symbol into slices with ranked rate and decoding complexity. The advantage over the existing carrier or time segmentation is that the proposed technique does not depend on the frequency channel to produce slices of equal rate. Also, the encoding and the decoding complexity is kept simple.
\end{abstract}

Index Terms - Orthogonal Frequency Division Multiplexing, Fast Fourier Transform

\section{INTRODUCTION}

Waveform flexibility is key in communication systems. Adaptive Code and Modulation, Software Defined Radio, waveform selection in cognitive radio, and the current radio slicing in fifth generation $(5 \mathrm{G})$ systems [1] are proof of it. This paper delves into the structure of the OFDM symbol [2] in order to gain in flexibility for its transmission, which can be instrumental for the needed flexibility in future $5 \mathrm{G}$ or next generation systems. OFDM has been extensively studied since the 90s as it was chosen for the physical layer transport in fourth-generation systems. For instance, references such as [3] and [4] introduce the concepts of multi-symbol encapsulation and time slicing in order to efficiently multiplex multimedia data streams with dynamic data rates. With the advent of $5 \mathrm{G}$, the term slice has been coined to also designate each of the different virtual networks over one physical network that deliver different services [5]. This has triggered interesting studies on multi-service signal multiplexing for radio access and physical layer slicing [6]. We note that the standard does not specify the strict values of the features that have to be guaranteed by a specific slice, which is left open for implementation. In its broader conception, they can be created either with more conventional time or carrier segmentation procedures as in [7],[8], which consider orthogonal multiplexing or, with more novel and general techniques such as those in [9] or [10]. Channelization techniques, as in [7], progressed a lot for software defined radios. They paved the way to the

Thanks to The Spanish ministry of Science, Innovation and Universities, under project TERESA - TEC2017-90093-C3-1-R (AEI/FEDER, UE). so-called bandwidth parts [8] in 5G, which orthogonally subdivide one OFDM carrier and can be used for different purposes, such as to support signals with different requirements so that the user terminals can optimize their resources (e.g., energy). In contrast, the alternatives in [9] or [10], multiplex mixed-numerology OFDM signals in a non-orthogonal way. The present work takes up the initial semantics of OFDM symbol slicing in [3] and [4] and proposes an orthogonal splitting of the OFDM symbol for a point to point transmission, with fixed carrier spacing. It is novel with respect to orthogonal frequency division multiple access (OFDMA), or the mentioned OFDM slicing techniques because it allows to successively obtain slices of equal rate irrespective of the frequency channel. Thus, it allows to scale down to the OFDM symbol the slicing policy. This paper focuses on the signal processing aspects of the proposed OFDM symbol splitting, and leaves for future work its evaluation and application to the suitable slice granularity by following, for instance, some of the plausible options that are identified in [11].

The signal processing presented in this work is motivated by the formalism used for polar codes, [12], and the extension of the concept of channel polarization. In fact, in [12], Erdal Arikan opened the door to use the Inverse Fast Fourier Transform/Fast Fourier Transform (IFFT/FFT) with binary algebra, due to the channel combining and channel splitting with the butterfly factor graph of the code. When working in the field of complex numbers, this channel combining process reminds of the manipulation of the modulated information symbols that OFDM carries out, which motivates our view that the polarization of a given channel in several subchannels is behind the well-known OFDM. Note that we are not conceiving channel polarization to construct capacity-achieving codes, but as transforming a given channel into channels with ranked reliability and decoding complexity. However, the IFFT/FFT only transforms a wideband channel into several narrowband ones and it does not provide any ranking of them, as they just follow the frequency response of the global channel. Perfect Channel State Information at the Transmitter (CSIT) is required in order to control the channel quality. This work proposes a technique that, applied to the OFDM symbol, overcomes the IFFT/FFT mentioned drawbacks and, without the need of CSIT, converts the channel into channels with ranked rate and decoding complexity. 
The following section 2 introduces the basic splitting of the channel and the transformation to be applied to an OFDM symbol in order to create different slices. Section 3 presents possible receivers and motivates an specific transform design in order to keep on using the simplicity of the FFT algorithm at reception. Finally, section 4 presents the general recursive architecture for the proposed orthogonal OFDM slicing, and illustrates its processing. Notation: Upper- and lower-case boldface letters denote matrices and vectors, respectively. Let the superscripts $(.)^{\mathrm{T}}$ and $(.)^{\mathrm{H}}$ denote transpose and Hermitian transpose operations, respectively. By $\mathbf{I}_{N}$ we denote the $N$-th order identity matrix. $\operatorname{diag}(\mathbf{a})$ is a diagonal matrix with the entries of the vector a on its diagonal.

\section{THE BASIC CHANNEL SPLITTING}

We consider the baseband model in (1) for the transmission of an OFDM symbol with $N$ narrowband subcarriers through a channel whose finite impulse response (FIR) is modeled with the coefficients $h_{n}, n=0, L-1$,

$$
\mathbf{y}=\mathbf{H}_{Z N} \mathbf{T}_{N} \mathbf{s}+\mathbf{w},
$$

where $\mathbf{s} \in C^{N \times 1}$ contains the OFDM time samples after the IFFT, $\mathbf{w} \in C^{N \times 1}$ is the additive white Gaussian noise with zero mean and variance equal to $\sigma^{2}, \mathbf{y} \in C^{N \times 1}$ contains the baseband time samples at the receiver once the Cyclic Prefix (CP) has been removed. We assume that the duration of the $\mathrm{CP}$ is larger than the delay spread of the channel [2], and that $\mathbf{H}_{Z N} \in C^{N \times N}$ is the effective time channel matrix, which is circulant. Time and frequency synchronization are assumed. $\mathbf{T}_{N} \in C^{N \times N}$ is the orthonormal transform that we incorporate in order to split the OFDM symbol into slices with desirable properties. Its formulation is

$$
\mathbf{T}_{N}=\frac{1}{\sqrt{2}}\left(\begin{array}{cc}
\mathbf{I}_{N / 2} & \mathbf{W}_{N / 2} \\
\mathbf{I}_{N / 2} & -\mathbf{W}_{N / 2}
\end{array}\right)
$$

where $N=2^{k}$ for $k$ integer, and matrix $\mathbf{W} \in C^{N / 2 \times N / 2}$ is an orthonormal matrix, which is further detailed in section 3 . Note that

$$
\mathbf{T}_{2}=\frac{1}{\sqrt{2}}\left(\begin{array}{cc}
1 & 1 \\
1 & -1
\end{array}\right),
$$

and that $\mathbf{T}_{1}=1$; thus, translating to the field of real numbers the generation matrix of size 2 that is used in polar codes, namely, $\left(\begin{array}{ll}1 & 1 \\ 1 & 0\end{array}\right)$. Concerning the channel matrix $\mathbf{H}_{Z N}$, whenever the channel is of length $L \leq N / 2$, it can be formulated as

$$
\mathbf{H}_{Z N}=\left(\begin{array}{cc}
\mathbf{H}_{N / 2} & \mathbf{H}_{C N / 2} \\
\mathbf{H}_{C N / 2} & \mathbf{H}_{N / 2}
\end{array}\right),
$$

where $\mathbf{H}_{N / 2} \in C^{N / 2 \times N / 2}$ is the following lower triangular and Toeplitz matrix

$$
\mathbf{H}_{N / 2}=\left(\begin{array}{cccc}
h_{0} & 0 & \ldots & 0 \\
h_{1} & h_{0} & \ldots & 0 \\
\ldots & \ldots & \ldots & \ldots \\
0 & h_{L-1} & \ldots & h_{0}
\end{array}\right) .
$$

$\mathbf{H}_{C N / 2} \in C^{N / 2 \times N / 2}$ is the circular complement of $\mathbf{H}_{N / 2}$,

$$
\mathbf{H}_{C N / 2}=\left(\begin{array}{cccccc}
0 & \ldots & h_{L-1} & \ldots & h_{2} & h_{1} \\
0 & \ldots & 0 & h_{L-1} & \ldots & h_{2} \\
\ldots & \ldots & \ldots & \ldots & \ldots & h_{L-1} \\
\ldots & \ldots & \ldots & \ldots & \ldots & \ldots \\
0 & \ldots & 0 & 0 & \ldots & 0
\end{array}\right) .
$$

Note that for $L \leq N / 2$ this circular complement matrix is strictly upper triangular. At the receiver, the corresponding transform pair, $\mathbf{T}_{N}^{H}$, is applied

$$
\mathbf{z}=\mathbf{T}_{N}^{H} \mathbf{y}=\mathbf{T}_{N}^{H} \mathbf{H}_{Z N} \mathbf{T}_{N} \mathbf{s}+\mathbf{T}_{N}^{H} \mathbf{w}=\mathbf{H}_{Z N}^{e} \mathbf{s}+\mathbf{T}_{N}^{H} \mathbf{w} .
$$

Next we show that the resulting equivalent channel, $\mathbf{H}_{Z N}^{e}$ splits into the so-called polarized channels. By operating $\mathbf{T}_{N}^{H} \mathbf{H}_{Z N} \mathbf{T}_{N}$ using (2) and (4), $\mathbf{H}_{Z N}^{e}$ can be formulated as:

$$
\begin{array}{cc}
\left(\begin{array}{cc}
\mathbf{H}_{N / 2}+\mathbf{H}_{C N / 2} & \mathbf{0}_{N / 2} \\
\mathbf{0}_{N / 2} & \mathbf{W}_{N / 2}^{H}\left(\mathbf{H}_{N / 2}-\mathbf{H}_{C N / 2}\right) \mathbf{W}_{N / 2}
\end{array}\right)= \\
=\left(\begin{array}{cc}
\mathbf{H}_{Z N / 2}^{+} & \mathbf{0}_{N / 2} \\
\mathbf{0}_{N / 2} & \mathbf{W}_{N / 2}^{H} \mathbf{H}_{Z N / 2}^{-} \mathbf{W}_{N / 2}
\end{array}\right) .
\end{array}
$$

Thus, the channel has been split into two polarized channels or physical layer slices. We denote the positive polarized channel as $\mathbf{H}_{Z N / 2}^{+}=\mathbf{H}_{N / 2}+\mathbf{H}_{C N / 2}$ and the basic negative polarized channel as $\mathbf{H}_{Z N / 2}^{-}=\mathbf{H}_{N / 2}-\mathbf{H}_{C N / 2}$. We call polarization or slicing step to the operation in (8) because from the transmitted $N$ samples it produces two new channels of size $N / 2$ with a mutual information $(M I)$ and a decoding complexity that can be ranked. This is explained in next subsections. We note that, differently to the polar codes, the advantage of the so-called polarization transformation $\mathbf{T}_{N}$ is not to achieve capacity or reduce the bit error rate, but to transform the time channel in OFDM into channels with ranked $M I$. On the one hand, matrix $\mathbf{H}_{Z N / 2}^{+}$preserves the circular symmetry and, whenever $L \leq N / 4$, it can be decomposed again into the previously introduced block matrices, now of size $N / 4$

$$
\mathbf{H}_{Z N / 2}^{+}=\left(\begin{array}{cc}
\mathbf{H}_{N / 4} & \mathbf{H}_{C N / 4} \\
\mathbf{H}_{C N / 4} & \mathbf{H}_{N / 4}
\end{array}\right) .
$$

Therefore, we could apply again the proposed transformation to this equivalent channel in order to further split it into two polarized channels. As $\mathbf{H}_{Z N / 2}^{+} \in C^{N / 2 \times N / 2}$, the transformation to be applied now is $\mathbf{T}_{N / 2}$. This feature can be exploited to recursively slice the OFDM symbol. Therefore, we are mimicking, within the complex field, the construction of polar codes, which relies on the recursive application of a linear transformation. On the other hand, the basic negative polarized $\mathbf{H}_{Z N / 2}^{-}$does not preserve the circular symmetry:

$$
\mathbf{H}_{Z N / 2}^{-}=\left(\begin{array}{cc}
\mathbf{H}_{N / 4} & -\mathbf{H}_{C N / 4} \\
\mathbf{H}_{C N / 4} & \mathbf{H}_{N / 4}
\end{array}\right) .
$$


Therefore, applying the transform pair $\left(\mathbf{T}_{N / 2}, \mathbf{T}_{N / 2}^{H}\right)$ to $\mathbf{W}_{N / 2}^{H} \mathbf{H}_{Z N / 2}^{-} \mathbf{W}_{N / 2}$ does not always split it into a positive and a negative polarized channel. Next, we study the $M I$ of these channels.

\subsection{Splitting of the Mutual Information}

Since the used transformation is orthonormal, the $M I$, in [bits/s/Hz], remains the same after its application. In other words, the $M I$ of $\mathbf{H}_{Z N}$ is the same as that of $\mathbf{T}_{N}^{H} \mathbf{H}_{Z N} \mathbf{T}_{N}$. For the purpose of physical layer slicing it is of interest to know the mutual information of each slice, namely, that of the positive and of the negative polarized channels, respectively. Let us consider uniform power allocation, $P$, and formulate the MI of the equivalent channel in (8) as

$$
M I=\log \operatorname{det}\left(\mathbf{I}_{N}+\frac{P}{\sigma^{2}} \mathbf{T}_{N}^{H} \mathbf{H}_{Z N} \mathbf{H}_{Z N}^{H} \mathbf{T}_{N}\right) .
$$

Due to the block diagonal structure of (8) and the orthonormal nature of $\mathbf{T}_{N}$, (11) can be decomposed into

$$
\begin{gathered}
M I=M I^{+}+M I^{-} \\
M I^{+}=\log \operatorname{det}\left(\mathbf{I}_{N / 2}+\frac{P}{\sigma^{2}} \mathbf{H}_{Z N / 2}^{+} \mathbf{H}_{Z N / 2}^{+H}\right) \\
M I^{-}=\log \operatorname{det}\left(\mathbf{I}_{N / 2}+\frac{P}{\sigma^{2}} \mathbf{W}_{N / 2}^{H} \mathbf{H}_{Z N / 2}^{-} \mathbf{H}_{Z N / 2}^{-H} \mathbf{W}_{N / 2}\right)
\end{gathered}
$$

To compare these expressions, the block decomposition in (9) and (10) can be introduced into (13) and (14), respectively (see [13] for more details). The result is that the difference between $M I^{+}$and $M I^{-}$is negligible if $L \ll N / 8$. In this case, the proposed transform allows to split one OFDM symbol of $N$ subcarriers into two slices of size $N / 2$ that approximately share half of the $M I$ each, irrespective of the channel $h(n)$. This is the key concept of the proposed slicing technique. Section 4 explains its implementation by recursively applying the proposed transform to create, within one OFDM symbol, more than 2 orthogonal slices with ranked rate.

\section{RECEIVER FOR THE POLARIZED CHANNELS}

Due to the block diagonal structure of the equivalent channel $\mathbf{H}_{Z N}^{e}$ in (8), we can deal with the transmission and reception of the symbols in $\mathbf{s}$ in two separate blocks or slices of size $N / 2$ each, $\mathbf{s}=\left[\mathbf{s}_{1}, \mathbf{s}_{2}\right]^{T}$, generated by two separate $N / 2$ IFFT. Namely, we can rewrite (7) as:

$$
\mathbf{z}=\left(\begin{array}{l}
\mathbf{z}_{1} \\
\mathbf{z}_{2}
\end{array}\right)=\left(\begin{array}{c}
\mathbf{H}_{Z N / 2}^{+} \\
\mathbf{W}_{N / 2}^{H} \mathbf{H}_{Z N / 2}^{-} \mathbf{W}_{N / 2}
\end{array}\right)\left(\begin{array}{l}
\mathbf{s}_{1} \\
\mathbf{s}_{2}
\end{array}\right)+\mathbf{T}_{N}^{H} \mathbf{w} .
$$

Since the positive polarized channel preserves the circular structure, the decoding of $\mathbf{z}_{1}$ is as in OFDM, i.e. $N / 2$-FFT and per symbol equalization with the frequency response of the positive polarized channel, assumed known at reception. This is not the case for the decoding of $\mathbf{z}_{2}$, because the negative polarized channel does not preserve circularity. Therefore, in general, it is needed a direct decoding with iterative techniques, such as, for instance, successive interference cancellation. In order to circumvent this problem and facilitate the decoding, we propose the design in (16) for the submatrix $\mathbf{W}_{N / 2}$ within $\mathbf{T}_{N}$

$$
\mathbf{W}_{N / 2}=\left(\begin{array}{cc}
\boldsymbol{\Omega}_{N / 4} & \mathbf{0}_{N / 4} \\
\mathbf{0}_{N / 4} & j \boldsymbol{\Omega}_{N / 4}
\end{array}\right),
$$

where $\boldsymbol{\Omega}_{N / 4}$ is the complete first step of a time-decimated IFFT [14]:

$$
\boldsymbol{\Omega}_{N / 4}=\operatorname{diag}\left(\exp \left(j 2 \pi \frac{m}{N} ; m=0, \ldots, \frac{N}{4}-1\right)\right) .
$$

If $L \leq N / 4$ then the negative polarized channel

$$
\begin{gathered}
\mathbf{W}_{N / 2}^{H} \mathbf{H}_{Z N / 2}^{-} \mathbf{W}_{N / 2}= \\
\left(\begin{array}{cc}
\boldsymbol{\Omega}_{N / 4}^{H} \mathbf{H}_{N / 4} \boldsymbol{\Omega}_{N / 4} & -j \boldsymbol{\Omega}_{N / 4}^{H} \mathbf{H}_{C N / 4} \boldsymbol{\Omega}_{N / 4} \\
-j \boldsymbol{\Omega}_{N / 4}^{H} \mathbf{H}_{C N / 4} \boldsymbol{\Omega}_{N / 4} & \boldsymbol{\Omega}_{N / 4}^{H} \mathbf{H}_{N / 4} \boldsymbol{\Omega}_{N / 4}
\end{array}\right),
\end{gathered}
$$

is circulant. Therefore, to decode each of the symbols contained in $\mathbf{z}_{2}$, a $N / 2$-FFT and per symbol equalization with the frequency response of the negative polarized channel can be applied. Let us note that, if the length of the FIR of the channel, $L$ is smaller than the slice size (i.e., $L \leq N / 2$ ), the frequency response of the positive and negative polarized channels in (15) corresponds to the even and odd terms of $H(l), l=0, \ldots, N-1$, respectively, where

$$
H(l)=\sum_{n=0}^{\frac{N}{2}-1} h(n) \exp \left(-j \ln \frac{2 \pi}{N}\right), n=0, \ldots, \frac{N}{2}-1 .
$$

Proof: $H(l)$ can be decomposed into its even and odd terms

$$
\begin{gathered}
H(l)=H(2 l)+H(2 l+1)= \\
=\sum_{n=0}^{\frac{N}{2}-1} h(n) \exp \left(-j \ln \frac{2 \pi}{N / 2}\right)+ \\
+\sum_{n=0}^{\frac{N}{2}-1} h(n) \exp \left(-j \frac{2 \pi}{N}\right) \exp \left(-j \ln \frac{2 \pi}{N / 2}\right) .
\end{gathered}
$$

The first term (21) is the frequency response of (9), or positive polarized channel. The second one, (22), is the frequency response of (18), or negative polarized channel.

If we analyze the decoding complexity of the proposed receiver, the $N / 2-$ FFT that is needed for $\mathbf{z}_{1}$ presents a complexity, in real operations, of $O\left(\frac{N}{2} \log _{2} \frac{N}{2}\right)$. The decoding of $\mathbf{z}_{2}$ requires the multiplication by $\mathbf{W}_{N / 2}^{H}$ before applying a 
$N / 2-$ FFT; thus, the complexity is $O\left(N+\frac{N}{2} \log _{2} \frac{N}{2}\right)$. Altogether, to decode $\mathbf{z}$ the complexity is the same as that of one $N$-FFT. The subsequent equalization, as studied, does not require more computation than in a regular OFDM transmission, because, as shown in (20) just an easy reordering of the frequency response before equalizing is needed. In conclusion, the advantage of the proposed transmission in (7) in front of the direct transmission of an OFDM symbol is that with the proposed scheme we are splitting the OFDM symbol into two slices of equal $M I$, irrespective of the channel.

\section{GENERAL RECURSIVE ARCHITECTURE}

If $\mathbf{H}_{Z N / 2}^{+}$keeps its circular structure (i.e., $L \leq N / 4$ ), the positive polarized channel can be sliced once more. In this way we obtain two slices of size $N / 4$ that rank in rate and decoding complexity; in other words, presenting a controlled QoS. For this purpose, the transformation $\mathbf{T}_{N / 2}$ is applied to $\mathbf{s}_{1}$ so that

$$
\mathbf{y}=\mathbf{H}_{Z N} \mathbf{T}_{N}\left(\begin{array}{c}
\mathbf{T}_{N / 2} \mathbf{s}_{1} \\
\mathbf{s}_{2}
\end{array}\right)+\mathbf{w}
$$

By substituting (2) into (23) we obtain (24).

$$
\begin{aligned}
\mathbf{y} & =\mathbf{H}_{Z N} \frac{1}{\sqrt{2}}\left(\begin{array}{cc}
\mathbf{T}_{N / 2} & \mathbf{W}_{N / 2} \\
\mathbf{T}_{N / 2} & -\mathbf{W}_{N / 2}
\end{array}\right)\left(\begin{array}{l}
\mathbf{s}_{1} \\
\mathbf{s}_{2}
\end{array}\right)+\mathbf{w} \\
& =\mathbf{H}_{Z N} \mathbf{T}_{N}^{R} \mathbf{s}+\mathbf{w}=\mathbf{H}_{Z N} \mathbf{x}_{T}+\mathbf{w} .
\end{aligned}
$$

Therefore, a recursive scheme with the orthonormal $\mathbf{T}_{N}^{R}$ is obtained by replacing $\mathbf{I}_{N / 2}$ in (2) by the transform $\mathbf{T}_{N / 2}$. The recursion implies twofold: i) at the transmitter $\mathbf{s}_{1}$ has to be generated by two $N / 4$-IFFT (see Fig. 1); ii) at the receiver $\mathbf{y}$ has to be multiplied by $\mathbf{T}_{N}^{R H}$ to obtain $\mathbf{s}$ plus noise of variance $\sigma^{2}$. In other words, it has to implement the mirrored structure of Fig. 1. Finally, to decode the information data, two $N / 4-$ FFT and one $N / 2-$ FFT are needed, resulting again the overall complexity of a $N-\mathrm{FFT}$, and 3 slices are obtained. In order to create one additional slice (i.e., a total of 4 slices), the same procedure can again be applied to the resulting $\mathbf{H}_{Z N / 4}^{+}$, whenever $L \leq N / 8$ (in practice, whenever the length of CP is $\leq N / 8$ ). This means that $\mathbf{T}_{N / 2}$ in (24) has to be replaced by $\mathbf{T}_{N / 2}^{R}$, and so on. Next, we illustrate this process by considering the ETU (Extended Typical Urban) channel, and $N=2048$ carriers. In the ETU channel, the last echo comes after $5000 \mathrm{~ns}$. If we consider a carrier spacing of $\Delta_{f}=15 \mathrm{KHz}$, which is prone to be used at sub-6 GHz transmission, the duration of the channel FIR is $L=155$ taps, and the CP 160 samples. The decoding process can be viewed in Fig. 2, where 3 slicing or polarization steps are carried out and 4 slices are produced. Note that the channel $\mathbf{H}_{Z N / 8}^{+}$is the one with the lowest decoding complexity. The next one is $\mathbf{W}_{N / 8}^{H} \mathbf{H}_{Z N / 8}^{-} \mathbf{W}_{N / 8}$ and so forth. Specifically, the number of total operations to decode the 4 slices is

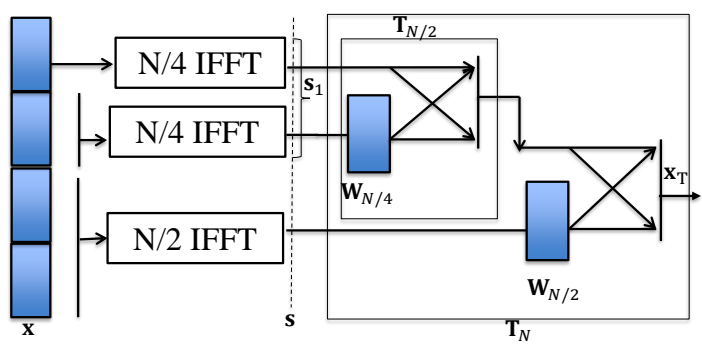

Fig. 1. Detailed transmitter design at baseband with 2 polarization steps to produce 3 slices.

22528 , which is the same as the FFT of the overall frame of length $N=2048$ (i.e., $N \log _{2}(N)=22528$ ). Figure 2 shows a uniform splitting of the $M I$ along the OFDM slicing process. Remarkably, the splitting can be uniform whenever the structure of the original channel is preserved. In other words $L \leq \frac{N}{2^{K}}$, where $K$ is the number of total polarization steps or transforms, which produce $K+1$ uniform slices. In this case $N=2048, L=155 \leq N / 2^{3}=N / 8=256$, and $K=3$ steps to obtain 4 slices. Further splitting can be done, but the $M I$ is not uniformly shared any more among the produced slices. For instance, in this example at sub- $6 \mathrm{GHz}$ and $\Delta_{f}=15$ $\mathrm{KHz}$, the small slices can be used for low-rate sensor data, [15]. The same kind of results can be obtained for other chan-

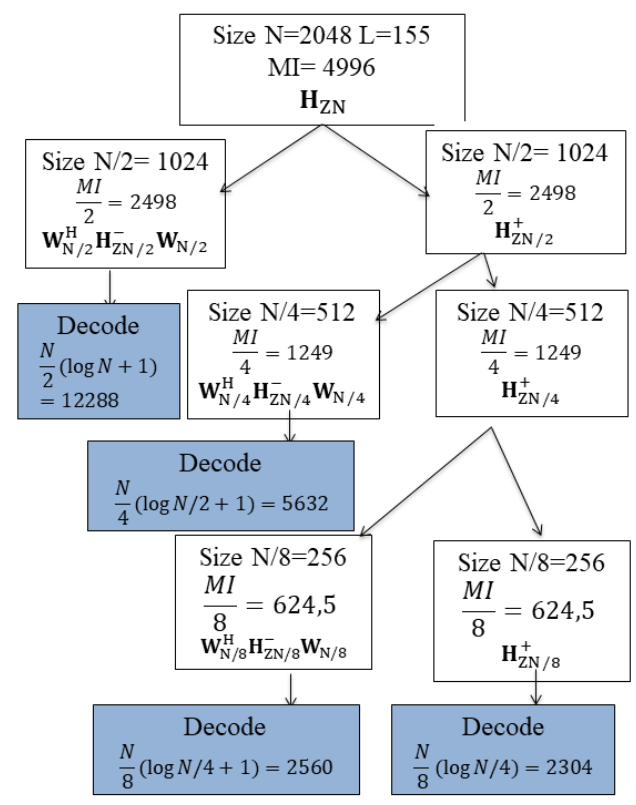

Fig. 2. Slice ranking: the resulting 4 slices (colored boxes) after 3 polarization transforms.

nels and numerology; validating the proposed transform to produce OFDM symbol slices with ranked rate of $\frac{M I}{2^{k}} \Delta_{f} N$ and decoding complexity. 


\section{REFERENCES}

[1] S. D’Oro, F. Restuccia, A. Talamonti, and T. Melodia, "The Slice is Served: Enforcing Radio Access Network Slicing in Virtualized 5G Systems," in Proceedings of IEEE International Conference on Computer Communications, April 2019.

[2] A. N. Akansu, P. Duhamel, Xueming Lin, and M. de Courville, "Orthogonal transmultiplexers in communication: a review," IEEE Transactions on Signal Processing, vol. 46, no. 4, pp. 979-995, 1998.

[3] X. Wang, Y. Wu, and J.Chouinard, "System design and implementation of multiple-symbol encapsulated ofdm," in Proceedings of the 2005 IEEE 61 st Vehicular Technology Conference. IEEE, 2005, vol. 2, pp. 10431047.

[4] X. Wang, Y. Wu, H. Wu, and J. Chouinard, "A time slicing technique for mobile multimedia communications using mse-ofdm system," in Proceedings of the IEEE Vehicular Technology Conference-Spring. IEEE, 2008, pp. 1231-1235.

[5] P. Popovski, O. Simeone K.F. Trillingsgaard, and G. Durisi, "5g wireless network slicing for embb, urlcc, and mmtc: A communication-theoretic view," in IEEE Access, 2018, pp. 55765-55779.

[6] K. Katsalis, N. Nikaein, E. Schiller, A. Ksentini, and T. Braun, "Network slices toward 5g communications: Slicing the lte network," IEEE Communications Magazine, vol. 55, no. 8, pp. 146-154, 2017.

[7] J.Yli-Kaakinen and M. Renfors, "Fast-convolution filter bank approach for non-contiguous spectrum use," in 2013 Future Network Mobile Summit, 2013, pp. 1-10.

[8] Sheri DeTomasi, "Understanding $5 \mathrm{~g}$ new radio bandwidth parts," in https://blogs.keysight.com/blogs/inds.entry.html/2018. Keysight, 2018.

[9] L. Zhang S. McWade, M. F. Flanagan and A. Farhang, "Interference and rate analysis of multinumerology noma," in Transactions on Wireless Communications. IEEE, 2020, pp. 5132-5147.

[10] O. Onireti P. Xiao M. A. Imran B. Yang, L. Zhang and R. Tafazolli, "Mixed-numerology signals transmission and interference cancellation for radio access network slicing," in IEEE Access, 2018, pp. 55765-55779.

[11] S. E. Elayoubi, S. B. Jemaa, Z. Altman, and A. GalindoSerrano, "5g ran slicing for verticals: Enablers and challenges," IEEE Communications Magazine, vol. 57, no. 1, pp. 28-34, 2019.
[12] E. Arikan, "Channel Polarization: A Method for Constructing Capacity-Achieving Codes for Symmetric Binary-Input Memoryless Channels," IEEE Transactions on Information Theory, vol. 55, no. 7, pp. 3051 -3073, april 2009.

[13] A. Perez and M.A. Lagunas, "Appendix mi slicing in ofdm," in www.cttc.es/wpcontent/uploads/2020/09/Appendix ${ }_{M} I$ Slicing.pdf.CTTC, 2020.

[14] E. Oran Brigham, “The Fast Fourier Transform," in Prentice-Hall, Inc., 1974.

[15] S. S. Lekshmi, M. S. Anjana, B. B. Nair, D. Raj, and S. Ponnekanti, "Framework for generic design of massive iot slice in 5g," in 2019 International Conference on Wireless Communications Signal Processing and Networking (WiSPNET), 2019, pp. 523-529. 